\title{
Existence and nonexistence of solutions for the generalized Camassa-Holm equation
}

\author{
Pan Xiujuan ${ }^{1,2 *}$, Shin Min Kang ${ }^{3}$ and Young Chel Kwun ${ }^{4}$
}

${ }^{\text {"Correspondence: }}$

panxiujuan@tjpu.edu.cn

1 School of Science, Tianjin

Polytechnic University, Tianjin

300387, China

${ }^{2}$ College of Management and

Economics, Tianjin University,

Tianjin 300072, China

Full list of author information is

available at the end of the article

\begin{abstract}
In this paper, we study the Cauchy problem of a generalized Camassa-Holm equation. It is shown that the equation is locally well posed when the initial data are sufficiently smooth. Moreover, we present a sufficient condition which guarantees the existence of low regularity solutions for the generalized Camassa-Holm equation by the method of energy estimate. Finally, the nonexistence of smooth solitary-wave solutions is investigated.
\end{abstract}

Keywords: generalized Camassa-Holm equation; energy estimate; low regularity solution; smooth solitary wave

MSC: 35Q51; 35Q53; 35B35

\section{Introduction}

Recently, Dullin et al. derived the following nonlinear partial differential equation [1]:

$$
m_{t}+c_{0} u_{x}+u m_{x}+2 m u_{x}=-\gamma u_{x x x}, \quad x \in \mathbb{R}, t \in \mathbb{R}
$$

by using asymptotic expansions directly in the Hamiltonian for Euler equations in the shallow water regime, and thereby it is shown to be bi-Hamiltonian and has a Lax pair formulation. Dullin-Gottwald-Holm (DGH) equation (1.1) models the unidirectional propagation of two-dimensional waves in shallow water over a flat bottom, where $u(t, x)$ stands for the horizontal component of the fluid velocity, $m=u-\alpha^{2} u_{x x}$ represents the momentum, $c_{0}=\sqrt{g h}$ (where $c_{0}:=2 \omega$ ) is the linear wave speed for undisturbed water at rest at spatial infinity, and the constants $\alpha^{2}$ and $\gamma / c_{0}$ are squares of length scales. DGH equation contains both the $\mathrm{KdV}$ equation (when $\alpha \rightarrow 0$ ) and the $\mathrm{CH}$ equation (when $\gamma \rightarrow 0$ ) as limiting cases and preserves integrability through the inverse scattering transform (IST) method. Thanks to $m=u-\alpha^{2} u_{x x}$, equation (1.1) will be reformulated as

$$
u_{t}-\alpha^{2} u_{x x t}+2 \omega u_{x}+3 u u_{x}+\gamma u_{x x x}=\alpha^{2}\left(2 u_{x} u_{x x}+u u_{x x x}\right), \quad t>0, x \in \mathbb{R}
$$

with the initial data $u_{0}(x)$.

Recently, Tian et al. [2] investigated the well-posedness of the Cauchy problem and the scattering problem for equation (1.2), studied the problem of passing to the limit as $\alpha \rightarrow 0$, and obtained the scattering data of the scattering problem. Li and Olver [3] studied the well-posedness, blow-up and the low regular solutions for an integrable nonlinearly dispersive model wave equation. Mustafa [4] investigated the low regularity conditions 
needed for the Cauchy problem of DGH equation via the semigroup approach of quasilinear hyperbolic equations of evolution and the viscosity method. Constantin and Lenells [5] presented a simple algorithm for the inverse scattering approach to the Camassa-Holm equation. Liu [6] and Zhou [7] investigated the problems of the existence of global solutions and the formation of singularities for the DGH equation. Tian et al. [8] studied the limit behavior of the solutions to a class of nonlinear dispersive wave equations. Ai and Gui [9] presented an algorithm for the inverse scattering problem associated to the Dullin-Gottwald-Holm equation and derived a sufficient condition which guarantees the existence of low regularity solutions for the generalized Dullin-Gottwald-Holm equation.

In this paper, we are interested in the Cauchy problem for the following generalized Camassa-Holm equation:

$$
\left\{\begin{array}{l}
u_{t}-\alpha^{2} u_{x x t}+2 \omega u_{x}+\beta u^{m} u_{x}+\gamma u_{x x x}=\alpha^{2}\left(2 u_{x} u_{x x}+u u_{x x x}\right), \quad t>0, x \in \mathbb{R} \\
u(0, x)=u_{0}(x)
\end{array}\right.
$$

where $m \in \mathbb{N}, m \geq 1$ and $\beta \neq 0$ are given. Observe that if $m=1, \beta=3$ and $\omega=\gamma=0$, then (1.3) is the classical $\mathrm{CH}$ equation.

Equation (1.3) was investigated in [10] and [11], where the local well-posedness problem was studied with the initial data $u_{0} \in H^{s}(\mathbb{R})$ for $s>\frac{3}{2}$. In particular, Ai et al. [12] studied the low regularity solutions for the generalized Camassa-Holm equation when $m=2$ in (1.3).

Motivated by [3,12] and [2], in this paper we aim to study the low regularity solutions and nonexistence of smooth solitary waves for the generalized Camassa-Holm equation (1.3) for the general case $m \in \mathbb{N}, m \geq 1$. For the sake of convenience, we always assume $\alpha=1$ in what follows.

\section{Notations}

We shall use the notation $\|\bullet\|_{L^{p}}$ for the norm of the space $L^{p}(\mathbb{R}), 1 \leq p<\infty$, i.e., $\|f\|_{L^{p}}=$ $\left(\int_{\mathbb{R}}|f|^{p} d x\right)^{1 / p}$. The space $L^{\infty}=L^{\infty}(\mathbb{R})$ consists of all essentially bounded, Lebesgue measurable functions with the norm $\|f\|_{\infty}=\inf _{m(e)=0} \sup _{x \in \mathbb{R} \backslash e}|f(x)|$. And we denote the norm in the Sobolev space $H^{s}=H^{s}(\mathbb{R})$ by

$$
\|f\|_{H^{s}}=\left(\int_{\mathbb{R}}\left(1+|\xi|^{2}\right)^{s}|\hat{f}(\xi)|^{2} d \xi\right)^{1 / 2}
$$

for $s \in \mathbb{R}$, where $\hat{f}(\xi)$ is the Fourier transform of $f(x)$. The operator $\Lambda_{\alpha}^{s}$ for any $\alpha, s \in \mathbb{R}$ means $\Lambda_{\alpha}^{s}=\left(1-\alpha^{2} \partial_{x}^{2}\right)^{\frac{s}{2}}$; in particular, $\Lambda^{s}:=\Lambda_{1}^{s}=\left(1-\partial_{x}^{2}\right)^{\frac{s}{2}}, \Lambda=\Lambda_{1}^{1}$.

The remainder of the paper is organized as follows. In Section 2, we investigate the local well-posedness of the generalized Camassa-Holm equations. Next, we present some results of the regularized generalized Camassa-Holm equations; from this, the low regularity solutions for the generalized Camassa-Holm equation (1.3) are studied in Section 3. Finally, in Section 4, we demonstrate the nonexistence of smooth solitary-wave solutions.

\section{Existence of strong solutions}

Note that if $p(x):=\frac{1}{2} e^{-|x|}, x \in \mathbb{R}$, then $\left(1-\partial_{x}^{2}\right)^{-1} f=p * f$ for all $f \in L^{2}(\mathbb{R})$ and $p *\left(u-u_{x x}\right)=u$, where $*$ denotes convolution with respect to the spatial variable $x$. Therefore, equation 
(1.3) (with $\alpha=1$ ) can be rewritten as the following equivalent form:

$$
\begin{cases}u_{t}+u u_{x}+\gamma u_{x}+\partial_{x} p *\left((2 \omega+\gamma) u+\frac{\beta}{(m+1)} u^{m+1}+\frac{1}{2} u_{x}^{2}-\frac{1}{2} u^{2}\right)=0, & t>0, x \in \mathbb{R}, \\ u(0, x)=u_{0}(x), & x \in \mathbb{R} .\end{cases}
$$

In [10], the following local well-posedness result was obtained (with a slight modification).

Theorem 2.1 Let $u_{0} \in H^{s}(\mathbb{R})$ with $s>\frac{3}{2}$. There exists a time $T>0$ such that the initialvalue problem (2.1) has a unique solution $u \in \mathcal{C}\left([0, T] ; H^{s}\right) \cap \mathcal{C}^{1}\left([0, T] ; H^{s-1}\right)$ and the map $u_{0} \mapsto u$ is continuous from the neighborhood of $u_{0}$ in $H^{s}$ into $\mathcal{C}\left([0, T] ; H^{s}\right) \cap \mathcal{C}^{1}\left([0, T] ; H^{s-1}\right)$. Moreover, the following Hamiltonian functionals are conserved:

$$
\frac{d}{d t} \int_{\mathbb{R}} u d x=0, \quad \frac{d}{d t} \int_{\mathbb{R}}\left(u^{2}+u_{x}^{2}\right) d x=0 \quad \text { for all } t \in[0, T] .
$$

We are now in a position to state a blow-up criterion for the generalized $\mathrm{CH}$ equation (2.1).

Theorem 2.2 Let $u_{0} \in H^{s}(\mathbb{R})$ with $s>\frac{3}{2}$, and $u$ be the corresponding solution to (2.1) in Theorem 2.1. Assume that $T^{*}>0$ is the maximal time of existence. Then

$$
T^{*}<\infty \Rightarrow \int_{0}^{T^{*}}\left\|\partial_{x} u\right\|_{L^{\infty}} d \tau=\infty
$$

Remark 2.3 The blow-up criterion (2.3) implies that the lifespan $T_{u_{0}}^{*}$ does not depend on the regularity index $s$ of the initial data $u_{0}$. Indeed, let $u_{0}$ be in $H^{s}$ for some $s>\frac{3}{2}$ and consider some $s^{\prime} \in\left(\frac{3}{2}, s\right)$. Denote by $u_{s}$ (resp., $u_{s^{\prime}}$ ) the corresponding maximal $H^{s}$ (resp., $H^{s^{\prime}}$ ) solution given by the above theorem. Denote by $T_{s}^{*}$ (resp., $T_{s^{\prime}}^{*}$ ) the lifespan of $u_{s}$ (resp., $u_{s^{\prime}}$. Since $H^{s} \hookrightarrow H^{s^{\prime}}$, uniqueness ensures that $T_{s}^{*} \leq T_{s^{\prime}}^{*}$ and that $u_{s} \equiv u_{s^{\prime}}$ on $\left[0, T_{s}^{*}\right)$. Now, if $T_{s}^{*}<T_{s^{\prime}}^{*}$, then we must have $u_{s^{\prime}}$ in $\mathcal{C}\left(\left[0, T_{s}^{*}\right] ; H^{s^{\prime}}\right)$. Hence, $u_{s^{\prime}} \in L^{1}\left(\left[0, T_{s}^{*}\right] ; W^{1, \infty}\right)$ by using the Sobolev embedding $H^{s^{\prime}}(\mathbb{R}) \hookrightarrow W^{1, \infty}(\mathbb{R})$, which contradicts the above blow-up criterion (2.3). Therefore, $T_{s}^{*}=T_{s^{\prime}}^{*}$.

\section{Existence of low regularity solutions}

Firstly, we investigate the following regularized equation (3.1):

$$
\left\{\begin{array}{l}
u_{t}-u_{x x t}+\varepsilon u_{x x x x t}+\left(2 \omega u+\frac{\beta}{m+1} u^{m+1}\right)_{x}+\gamma u_{x x x}=2 u_{x} u_{x x}+u u_{x x x}, \quad t>0, x \in \mathbb{R}, \\
u(0, x)=u_{0}(x) .
\end{array}\right.
$$

By using the contraction mapping principle, we may easily obtain the following existence result, and we omit its proof here.

Theorem 3.1 Suppose that $u_{0} \in H^{s}(\mathbb{R})$ with $s \geq 1$, there exists $T>0$ depending only on $\left\|u_{0}\right\|_{H^{s}}$ such that there corresponds a unique solution $u(t, x) \in C\left([0, T] ; H^{s}\right)$ of equation (3.1) in the sense of distribution. If $s \geq 2$, the solution $u(t, x) \in C\left([0, \infty) ; H^{s}\right)$ exists globally in time. In particular, for $s \geq 4$, the corresponding solution is a classical globally defined solution of (3.1). 
Thanks to standard energy estimates, we have the following theorem.

Theorem 3.2 Let $s \geq 4$ and the function $u(x, t)$ be a solution of the regularized equation (3.1) with the initial data $u_{0}(x) \in H^{s}$. Then the following inequalities hold:

$$
\|u\|_{H^{1}}^{2} \leq c \int_{\mathbb{R}}\left(u^{2}+u_{x}^{2}+\varepsilon u_{x x}^{2}\right) d x=c \int_{\mathbb{R}}\left(u_{0}^{2}+u_{0 x}^{2}+\varepsilon u_{0 x x}^{2}\right) d x .
$$

For any $q \in(0, s-1]$, there is a constant $c$, depending only on $q$ and $m$, such that

$$
\|u\|_{H^{q+1}}^{2} \leq \int_{\mathbb{R}}\left(\left(\Lambda^{q+1} u_{0}\right)^{2}+\varepsilon\left(\Lambda^{q} u_{0 x x}\right)^{2}\right) d x+c \int_{0}^{t}\left\|u_{x}\right\|_{L^{\infty}}\|u\|_{L^{\infty}}^{m-1}\|u\|_{H^{q+1}}^{2} d \tau
$$

For any $q \in\left(\frac{1}{2}, s-1\right]$ and any $r \in\left(\frac{1}{2}, q\right]$, there is a constant $c$, depending only on $r, q$ and $m$, such that

$$
\|u\|_{H^{q+1}}^{2} \leq \int_{\mathbb{R}}\left(\left(\Lambda^{q+1} u_{0}\right)^{2}+\varepsilon\left(\Lambda^{q} u_{0 x x}\right)^{2}\right) d x+c \int_{0}^{t}\|u\|_{H^{r+1}}\|u\|_{H^{r}}^{m-1}\|u\|_{H^{q+1}}^{2} d \tau
$$

For any $q \in[0, s-1]$ and any $r \in\left(\frac{1}{2}, q\right]$, there is a constant $c$, independent of $\varepsilon$, such that

$$
(1-2 \varepsilon)\left\|u_{t}\right\|_{H^{q}} \leq c\|u\|_{H^{q+1}}\left(1+\|u\|_{L^{\infty}}^{m-1}\|u\|_{H^{1}}\right)
$$

Proof The proof can be similarly treated as in [3] or [8], and we omit it.

We recall the following lemma, which is critical to the proof of Theorem 3.4.

Lemma 3.3 ([3]) Let $u_{\varepsilon 0}$ be the convolution $u_{\varepsilon 0}=\phi_{\varepsilon} * u_{0}$ of the functions $\phi_{\varepsilon}(x)=$ $\varepsilon^{-1 / 4} \phi\left(\varepsilon^{-1 / 4} x\right)$ and $u_{0}$ such that the Fourier transform $\hat{\phi}$ of $\phi$ satisfies $\hat{\phi} \in C_{c}^{\infty}, \hat{\phi}(\xi) \geq 0$, and $\hat{\phi}(\xi)=1$ for any $\xi \in(-1,1)$. The following estimates hold for any $\varepsilon$ with $0<\varepsilon<1 / 4$ :

$$
\begin{aligned}
& \left\|u_{\varepsilon 0}\right\|_{H^{q}} \leq c, \quad\left\|u_{\varepsilon 0}-u_{0}\right\|_{H^{q}} \leq c \varepsilon^{\frac{s-q}{4}} \quad(q \leq s), \\
& \left\|u_{\varepsilon 0}\right\|_{H^{q}} \leq c \varepsilon^{\frac{s-q}{4}} \quad(q>s), \quad\left\|u_{\varepsilon 0}-u_{0}\right\|_{H^{s}}=o(1) .
\end{aligned}
$$

We shall show that some norms of the weak solution to the generalized $\mathrm{CH}$ equation (1.3) are bounded when $\varepsilon$ is sufficiently small.

Theorem 3.4 Suppose that $u_{0} \in H^{s}(\mathbb{R})$ for some $1 \leq s \leq \frac{3}{2}$ such that $\left\|u_{0 x}\right\|_{L^{\infty}}<\infty$. Let $u_{0 \varepsilon}$ be defined as in Lemma 3.3. Then there exist constants $T>0$ and $c>0$ independent of $\varepsilon$ such that the corresponding solution $u_{\varepsilon}$ of (3.1) satisfies $\left\|u_{\varepsilon x}\right\|_{L^{\infty}} \leq c$.

Proof We first study the regularized equation (3.1) with $u=u_{\varepsilon}$. Differentiating the first equation of (3.1) with respect to $x$ yields

$$
\begin{aligned}
& (1-\varepsilon) u_{x t}-\varepsilon u_{x x x t}-\gamma u_{x x}-(\gamma+2 \omega) u-\frac{\beta}{m+1} u^{m+1}+\frac{1}{2}\left(u^{2}\right)_{x x}-\frac{1}{2} u_{x}^{2}+\frac{1}{2} u^{2} \\
& \quad=-\Lambda^{-2}\left[\varepsilon u_{x t}+(2 \omega+\gamma) u+\frac{\beta}{m+1} u^{m+1}+\frac{u_{x}^{2}}{2}-\frac{1}{2} u^{2}\right] .
\end{aligned}
$$


Let $p \geq 1$ be an integer. Multiplying equation (3.1) by $\left(u_{x}\right)^{2 p+1}$, and then integrating the resulting equation with respect to $x$, we get

$$
\begin{gathered}
\frac{1-\varepsilon}{2 p+2} \frac{d}{d t} \int_{\mathbb{R}}\left(u_{x}\right)^{2 p+2} d x+\int_{\mathbb{R}}\left(u_{x}\right)^{2 p+1}\left(-\gamma u-2 \omega u-\frac{\beta}{m+1} u^{m+1}+\frac{1}{2} u^{2}\right) d x \\
+\frac{p}{2 p+2} \int_{\mathbb{R}}\left(u_{x}\right)^{2 p+3} d x-\int_{\mathbb{R}}\left(u_{x}\right)^{2 p+1} \varepsilon u_{x x x t} d x-\gamma \int_{\mathbb{R}}\left(u_{x}\right)^{2 p+1} u_{x x} d x \\
=-\int_{\mathbb{R}}\left(u_{x}\right)^{2 p+1} \Lambda^{-2}\left[\varepsilon u_{x t}+(2 \omega+\gamma) u+\frac{\beta}{m+1} u^{m+1}+\frac{u_{x}^{2}}{2}-\frac{1}{2} u^{2}\right] d x .
\end{gathered}
$$

Thanks to integration by parts and Hölder's inequality, we have

$$
\begin{aligned}
& \frac{1-\varepsilon}{2 p+2} \frac{d}{d t}\left\|u_{x}\right\|_{L^{2 p+2}}^{2 p+2} \\
& \quad \leq\left\|u_{x}\right\|_{L^{2 p+2}}^{2 p+1}\left\{\varepsilon\left\|u_{x x x t}\right\|_{L^{2 p+2}}+(\gamma+2 \omega)\|u\|_{L^{2 p+2}}\right. \\
& \left.\quad+\frac{\beta}{m+1}\left\|u^{m+1}\right\|_{L^{2 p+2}}+\frac{1}{2}\left\|u^{2}\right\|_{L^{2 p+2}}+\|g\|_{L^{2 p+2}}\right\}+\frac{p}{2 p+2}\left\|u_{x}\right\|_{L^{\infty}}\left\|u_{x}\right\|_{L^{2 p+2}}^{2 p+2},
\end{aligned}
$$

where

$$
g=\Lambda^{-2}\left[\varepsilon u_{x t}+(2 \omega+\gamma) u+\frac{\beta}{m+1} u^{m+1}+\frac{u_{x}^{2}}{2}-\frac{1}{2} u^{2}\right] .
$$

Because $\|f\|_{L^{p}} \rightarrow\|f\|_{L^{\infty}}$ as $p \rightarrow \infty$ for any $f \in L^{\infty} \cap L^{2}$, integrating (3.3) with respect to $t$ and then taking the limit as $p \rightarrow \infty$, we obtain

$$
\begin{aligned}
(1-\varepsilon)\left\|u_{x}\right\|_{L^{\infty}}(t) & \\
\leq & (1-\varepsilon)\left\|u_{0 x}\right\|_{L^{\infty}}+c \int_{0}^{t}\left[\varepsilon\left\|u_{x x x t}\right\|_{L^{\infty}}+\|u\|_{L^{\infty}}+\left\|u^{m+1}\right\|_{L^{\infty}}\right. \\
& \left.+\left\|u^{2}\right\|_{L^{\infty}}+\|g\|_{L^{\infty}}+\left\|u_{x}\right\|_{L^{\infty}}^{2}\right] d \tau .
\end{aligned}
$$

Applying the Sobolev imbedding theorem leads to

$$
\begin{gathered}
\left\|u^{m+1}\right\|_{L^{\infty}} \leq c\left\|u^{m+1}\right\|_{H^{1}} \leq c\|u\|_{H^{1}}^{m+1} \leq c_{1}, \\
\left\|u^{2}\right\|_{L^{\infty}} \leq c\left\|u^{2}\right\|_{H^{1}} \leq c\|u\|_{H^{1}}^{2} \leq c_{1},
\end{gathered}
$$

and

$$
\begin{aligned}
\|g\|_{L^{\infty}} & \leq\left\|\Lambda^{-2}\left[(2 \omega+\gamma) u+\frac{\beta}{m+1} u^{m+1}-\frac{1}{2} u^{2}\right]\right\|_{L^{\infty}}+\left\|\Lambda^{-2}\left(\varepsilon u_{x t}+\frac{u_{x}^{2}}{2}\right)\right\|_{H^{\frac{1}{2}}} \\
& \leq c_{1}+c_{2}\left(\left\|u_{t}\right\|_{L^{2}}+\left\|u_{x}\right\|_{L^{\infty}}\left\|u_{x}\right\|_{L^{2}}\right) \\
& \leq c_{1}+c_{2}\left(\left\|u_{t}\right\|_{L^{2}}+\left\|u_{x}\right\|_{L^{\infty}}^{2}\right),
\end{aligned}
$$

where we used the algebraic property of the Sobolev space $H^{s}(\mathbb{R})$ (with $s>\frac{1}{2}$ ), and constants $c_{1}$ and $c_{2}$ are independent of $\varepsilon$ with $\varepsilon$ sufficiently small. By (3.2), (3.4) and (3.5), we 
have

$$
\|g\|_{L^{\infty}} \leq c_{1}\left(c_{2}+\left\|u_{0 \varepsilon}\right\|_{H^{1}}^{2}\right) \leq c_{3}
$$

where the constant $c_{3}$ is independent of $\varepsilon$. Moreover, for any fixed $r \in\left(\frac{1}{2}, 1\right)$, there exists a constant $c_{r}$ such that $\left\|u_{x x x t}\right\|_{L^{\infty}} \leq c_{r}\left\|u_{x x x t}\right\|_{H^{r}} \leq c_{r}\left\|u_{t}\right\|_{H^{r+3}}$. Thanks to Theorem 3.2, one can see

$$
\left\|u_{x x x t}\right\|_{L^{\infty}} \leq c_{r}\|u\|_{H^{r+4}} .
$$

Thanks to Gronwall's inequality applied to (3.3) with $q=r+3$ and $u=u_{\varepsilon}$, we have

$$
\|u\|_{H^{r+4}}^{2} \leq \int_{\mathbb{R}}\left(\left(\Lambda^{r+4} u_{0}\right)^{2}+\varepsilon\left(\Lambda^{r+3} u_{0 x x}\right)^{2}\right) d x \exp \left(c \int_{0}^{t}\left\|u_{x}\right\|_{L^{\infty}} d \tau\right) .
$$

Therefore, thanks to (3.7), we get that for some constant $c$,

$$
\left\|u_{x x x t}\right\|_{L^{\infty}} \leq c \varepsilon^{\frac{s-r-4}{4}} \exp \left(c \int_{0}^{t}\left\|u_{x}\right\|_{L^{\infty}} d \tau\right)
$$

For $\varepsilon<\frac{1}{4}$, thanks to (3.6), (3.7), (3.8) and (3.9), we get

$$
\left\|u_{x}\right\|_{L^{\infty}}(t) \leq\left\|u_{0 x}\right\|_{L^{\infty}}+c \int_{0}^{t}\left[\varepsilon^{\frac{s-r}{4}} \exp \left(c \int_{0}^{\tau}\left\|u_{x}\right\|_{L^{\infty}} d s\right)+\frac{1}{2}\left\|u_{x}\right\|_{L^{\infty}}^{2}+1\right] d \tau .
$$

Hence, thanks to the contraction mapping principle applied, we obtain that there exists $T>0$ such that the integral equation

$$
f(t)=\left\|u_{0 x}\right\|_{L^{\infty}}+c \int_{0}^{t}\left[\varepsilon^{\frac{s-r}{4}} \exp \left(c \int_{0}^{\tau} f(s) d s\right)+\frac{1}{2} f^{2}(\tau)+1\right] d \tau
$$

has a unique solution $f(t) \in C([0, T])$. The comparison principle leads to the estimate $\left\|u_{x}\right\|_{L^{\infty}} \leq f(t)$ for any $t \in[0, T]$, which implies the conclusion of Theorem 3.4.

With Theorem 3.4 in hand, we can get the existence of a weak solution to the Cauchy problem (1.3) as follows.

Theorem 3.5 Suppose that $u_{0} \in H^{s}(\mathbb{R})$ for some $1 \leq s \leq \frac{3}{2}$ such that $\left\|u_{0 x}\right\|_{L^{\infty}}<\infty$. Then there exists a constant $T>0$ such that the Cauchy problem (1.3) has a solution $u(x, t) \in$ $L^{2}\left([0, T] ; H^{s}\right)$ in the sense of distribution and $u_{x} \in L^{2}([0, T] \times \mathbb{R})$.

Proof It follows from Theorem 3.4 that $\left\{u_{\varepsilon_{n} x}\right\}$ is bounded in the space $L^{\infty}$ with $\varepsilon_{n} \rightarrow 0$. Therefore, the sequences $\left\{u_{\varepsilon_{n}}^{m}\right\}$ and $\left\{u_{\varepsilon_{n} x}^{2}\right\}$ are weakly convergent to $u^{m}$ and $u_{x}^{2}$ in $L^{2}\left([0, T] ; H^{r}(-R, R)\right)$ for any $r \in[0, s-1)$, respectively. From this, we get, for any $f \in C_{c}^{\infty}$, that the solution $u$ satisfies the equation

$$
\begin{aligned}
& \int_{0}^{T} \int_{\mathbb{R}} u\left(f_{t}-f_{x x t}\right) d x d t \\
& \quad=\int_{0}^{T} \int_{\mathbb{R}}\left(\frac{u^{2}}{2} f_{x x x}-\gamma u f_{x x x}-\left(2 \omega u+\frac{\beta}{m+1} u^{m+1}+\frac{1}{2} u_{x}^{2}\right) f_{x}\right) d x d t,
\end{aligned}
$$


with $u(x, 0)=u_{0}$. Since $X=L^{1}([0, T] \times \mathbb{R})$ is a separable Banach space and $\left\{u_{\varepsilon_{n} x}\right\}$ is a bounded sequence in the dual space $X^{\star}=L^{\infty}([0, T] \times \mathbb{R})$ of $X$, there exists a subsequence of $\left\{u_{\varepsilon_{n} x}\right\}$, still denoted by $\left\{u_{\varepsilon_{n} x}\right\}$, weakly star convergent to a function $v$ in $L^{\infty}([0, T] \times \mathbb{R})$. From this and the fact that $\left\{u_{\varepsilon_{n} x}\right\}$ is also weakly convergent to $u_{x}$ in $L^{2}([0, T] \times \mathbb{R})$, we get that $u_{x}=v$ almost everywhere. Hence, $u_{x} \in L^{\infty}([0, T] \times \mathbb{R})$, and we end the proof of Theorem 3.5.

Remark 3.6 The proof of Theorem 3.5 is inspired by the argument of Ai et al. [12] in the study of the low regular solution to a generalized DGH equation. However, one problematic issue is that we here deal with the strong nonlinear term $u^{m}$ in our equation (1.3), making the proof of several required nonlinear estimates somewhat delicate. With the help of the method of weak convergence, this difficulty is nevertheless overcome by careful estimates for each approximate component of solutions.

\section{Nonexistence of smooth solitary waves}

It is observed that the peaked solution of the Camassa-Holm equation is not a smooth solution. Actually, one can establish the following result for any traveling wave solutions of the following generalized Camassa-Holm equation (with $m=1$ ):

$$
u_{t}-\alpha^{2} u_{x x t}+2 \omega u_{x}+\beta u u_{x}+\gamma u_{x x x}=\alpha^{2}\left(2 u_{x} u_{x x}+u u_{x x x}\right) .
$$

Theorem 4.1 There is no nontrivial traveling-wave solution $u \in C\left([0, \infty) ; H^{3}\right) \cap C^{1}([0, \infty)$; $H^{2}$ ) for equation (4.1) with $\gamma=-2 \omega \alpha^{2}$.

Proof Arguing by contradiction, assume that $w \in H^{3}$ and $u(t, x)=w(x-c t), c \neq 0$ is a strong solution of (4.1). Then we have

$$
c w^{\prime}-c \alpha^{2} w^{\prime \prime \prime}+2 \omega w^{\prime}+\gamma w^{\prime \prime \prime}-3 w w^{\prime}+2 \alpha^{2} w^{\prime} w^{\prime \prime}+\alpha^{2} w w^{\prime \prime \prime}=0 \quad \text { in } L^{2} .
$$

We find that

$$
\left(c w-c \alpha^{2} w^{\prime \prime}+2 \omega w+\gamma w^{\prime \prime}-\frac{3}{2} w^{2}+\frac{1}{2} \alpha^{2}\left(w^{\prime}\right)^{2}+\alpha^{2} w w^{\prime \prime}\right)^{\prime}=0 \quad \text { in } L^{2},
$$

and therefore

$$
c w-c \alpha^{2} w^{\prime \prime}+2 \omega w+\gamma w^{\prime \prime}-\frac{3}{2} w^{2}+\frac{1}{2} \alpha^{2}\left(w^{\prime}\right)^{2}+\alpha^{2} w w^{\prime \prime}=0 \quad \text { in } H^{1}
$$

or, what is the same (according to the fact $\gamma=-2 \omega \alpha^{2}$ ),

$$
\left(c-\frac{\gamma}{\alpha^{2}}-w\right)\left(w-\alpha^{2} w^{\prime \prime}\right)-\frac{1}{2}\left(w^{2}-\alpha^{2}\left(w^{\prime}\right)^{2}\right)=0 \quad \text { in } H^{1}
$$

since $w \in H^{3} \subset C_{0}^{2}(\mathbb{R})$. Multiplying this identity by $2 w^{\prime}$ yields that

$$
\left(c-\frac{\gamma}{\alpha^{2}}-w\right)\left(w^{2}-\alpha^{2}\left(w^{\prime}\right)^{2}\right)^{\prime}-w^{\prime}\left(w^{2}-\alpha^{2}\left(w^{\prime}\right)^{2}\right)=0 .
$$

Since $w \in H^{3} \subset C_{0}^{2}(\mathbb{R})$, we have $w \neq c$ a.e. and $w^{2} \neq \alpha^{2}\left(w^{\prime}\right)^{2}$ a.e. 
Let $w_{0}=w(\xi)=\max _{x \in \mathbb{R}} w(x)>0$. Then taking integration for $(4.2)$ in $[\xi, x]$ yields

$$
\int_{\xi}^{x} \frac{d\left(w^{2}-\alpha^{2}\left(w^{\prime}\right)^{2}\right)}{w^{2}-\alpha^{2}\left(w^{\prime}\right)^{2}}=\int_{\xi}^{x} \frac{d w}{c-\frac{\gamma}{\alpha^{2}}-w}, \quad x \in \mathbb{R} .
$$

This implies that

$$
\left|w+\frac{\gamma}{\alpha^{2}}-c\right|\left|w^{2}-\alpha^{2}\left(w^{\prime}\right)^{2}\right|=w_{0}^{2}\left|w_{0}+\frac{\gamma}{\alpha^{2}}-c\right|, \quad x \in \mathbb{R} .
$$

If we take into account $w, w^{\prime} \rightarrow 0$ as $x \rightarrow \infty$, it is then inferred from (4.3) that

$$
w_{0}^{2}\left|w_{0}+\frac{\gamma}{\alpha^{2}}-c\right|=0
$$

which also implies from (4.3) that

$$
\left|w+\frac{\gamma}{\alpha^{2}}-c\right|\left|w^{2}-\alpha^{2}\left(w^{\prime}\right)^{2}\right|=0, \quad x \in \mathbb{R} .
$$

This leads to a contradiction since $w \in H^{3}$.

Remark 4.2 Theorem 3.5 ensures that there exists a low regularity solution for (4.1). However, Theorem 4.1 asserts that it is not a solitary wave if it is smooth enough.

\section{Competing interests}

The authors declare that they have no competing interests.

\section{Authors' contributions}

All authors contributed equally to the writing of this paper. All authors read and approved the final manuscript.

\section{Author details}

${ }^{1}$ School of Science, Tianjin Polytechnic University, Tianjin 300387, China. ${ }^{2}$ College of Management and Economics, Tianjin University, Tianjin 300072, China. ${ }^{3}$ Department of Mathematics and the RINS, Gyeongsang National University, Jinju 660-701, Korea. ${ }^{4}$ Department of Mathematics, Dong-A University, Pusan 614-714, Korea.

\section{Acknowledgements}

The project is supported by National Natural Science Funds of China (Grant No. 11301480, 11371021).

Received: 17 February 2014 Accepted: 3 April 2014 Published:\#PUBLICATION_DATE

\section{References}

1. Dullin, R, Gottwald, G, Holm, DD: An integrable shallow water equation with linear and nonlinear dispersion. Phys. Rev. Lett. 87(9), 4501-4504 (2001)

2. Tian, L, Gui, G, Liu, Y: On the well-posedness problem and the scattering problem for the Dullin-Gottwald-Holm equation. Commun. Math. Phys. 257, 671-704 (2005)

3. Li, Y, Olver, P: Well-posedness and blow-up solutions for an integrable nonlinearly dispersive model wave equation. J. Differ. Equ. 162, 27-63 (2000)

4. Mustafa, OG: Existence and uniqueness of low regularity solutions for the Dullin-Gottwald-Holm equation. Commun. Math. Phys. 265, 189-200 (2006)

5. Constantin, A, Lenells, J: On the inverse scattering approach to the Camassa-Holm equation. J. Nonlinear Math. Phys. 10(3), 252-255 (2003)

6. Liu, Y: Global existence and blow-up solutions for a nonlinear shallow water equation. Math. Ann. 335, 717-735 (2006)

7. Zhou, Y: Blow-up of solutions to the DGH equation. J. Funct. Anal. 250, 227-248 (2007)

8. Tian, L, Gui, G, Guo, B: The limit behavior of the solutions to a class of nonlinear dispersive wave equations. J. Math. Anal. Appl. 341, 1311-1333 (2008)

9. $\mathrm{Ai}, \mathrm{X}, \mathrm{Gui}, \mathrm{G}$ : On the inverse scattering problem and the low regularity solutions for the Dullin-Gottwald-Holm equation. Nonlinear Anal., Real World Appl. 11(2), 888-894 (2010) 
10. Hakkaev, S, Kirchev, K: Local well-posedness and orbital stability of solitary wave solutions for the generalized Camassa-Holm equation. Commun. Partial Differ. Equ. 30, 761-781 (2005)

11. Hakkaev, S, Kirchev, K: On the well-posedness and stability of peakons for a generalized Camassa-Holm equation. Int. J. Nonlinear Sci. 1(3), 139-148 (2006)

12. Ai, X, Tian, L, Gui, G: On the low regularity solutions for the generalized Dullin-Gottwald-Holm equation. Int. J. Nonlinear Sci. 7(1), 113-118 (2009)

13. Gui, G, Liu, Y, Tian, L: Global existence and blow-up phenomena for the peakon b-family of equations. Indiana Univ. Math. J. 57(3), 1209-1234 (2008)

\#DIGITAL OBJECT IDENTIFIER

Cite this article as: Xiujuan et al.: Existence and nonexistence of solutions for the generalized Camassa-Holm equation. Advances in Difference Equations \#CITATION

Submit your manuscript to a SpringerOpen ${ }^{\circ}$ journal and benefit from:

- Convenient online submission

- Rigorous peer review

- Immediate publication on acceptance

Open access: articles freely available online

- High visibility within the field

- Retaining the copyright to your article

Submit your next manuscript at springeropen.com 